\title{
Research article \\ A prospective observational study on the traditional and novel risk factors associated with post-transplant diabetes mellitus in renal transplant recipients
}

\author{
Krithika Muralidhara ${ }^{1}$, Subramanian Kannan ${ }^{2}$, Ishthiaque Ahamed ${ }^{3}$, Krishna Kishore ${ }^{3}$, Llyod Vincent ${ }^{3}$, Nitin Hegde ${ }^{3}$ \\ ${ }^{1}$ Department of Nephrology, St. John's Medical College Hospital, Bangalore, India \\ ${ }^{2}$ Department of Endocrinology, ${ }^{3}$ Department of Nephrology, Narayana Hrudayalaya Hospitals Bangalore, India
}

(Received: March $2021 \quad$ Revised: June $2021 \quad$ Accepted: June 2021)

Corresponding author: Krithika Muralidhara. Email: krithidm@yahoo.co.in

\begin{abstract}
Introduction and Aim: Post Transplant Diabetes Mellitus (PTDM) constitutes a major co-morbidity that has significant impact on the patient and renal allograft outcome. Various traditional risk factors like dialysis vintage, immunosuppressive medication, viral infections have known to be associated with PTDM. Novel risk factors and its causative role in PTDM remains unexplored. The study looked at the incidence of PTDM at 6 months post renal transplantation and correlation of PTDM with known traditional and novel risk factors like pre-transplant Creactive protein (CRP) level and Trace element deficiency.
\end{abstract}

Material and Methods: 30 consecutive renal transplant recipients more than 18 years of age were inducted into the study. Demographic data, known pre-transplant and post-transplant risk factors associated with development of PTDM were collected. Pre-transplant CRP and serum Trace element (Aluminum, Copper, Selenium and Zinc) levels were estimated. PTDM was diagnosed by home based glucometer monitoring and confirmed by Oral glucose tolerance test (OGTT).

Results: The incidence of PTDM was 36.6\% at six months post renal transplant. Among the pre-transplant risk factors, higher age and positive family history of diabetes mellitus had a strong association with the occurrence of PTDM. Pre-transplant Zinc deficiency emerged as a significant novel risk factor for the development of PTDM.

Conclusion: Apart from known traditional risk factors, novel risk factors are associated with development of PTDM.

Keywords: Post Transplant Diabetes mellitus (PTDM); renal transplant; trace element; post-transplant transient Hyperglycemia (PTTH).

\section{INTRODUCTION}

$\mathrm{R}$ enal transplantation has now become the standard of care for End Stage Renal Disease (ESRD) resulting in better quality of life and improved patient survival by 10-15 years. However, it is associated with various complications and one among them is Post Transplant Diabetes Mellitus (PTDM). PTDM is defined according to the American Diabetes Association (ADA) criteria (1) and its occurrence has been described in up to $30 \%$ of kidney-transplant recipients (2). PTDM constitutes a major co-morbidity that has significant impact on the patient and allograft outcome. Several risk factors have been associated with the occurrence of PTDM. They include non-modifiable factors such as age, ethnicity, family history of diabetes mellitus and modifiable factors such as corticosteroids, calcineurin inhibitors, cytomegalovirus infection and hepatitis $\mathrm{C}$ viral infection (2). Beside these known risk factors, other risk factors like pre-transplant inflammatory status and Post-Transplant Transient Hyperglycemia (PTTH) have been studied. Few studies have looked at trace element deficiency leading to Type 2 diabetes mellitus in the general population. However, its causative role in PTDM remains unexplored. Therefore, this prospective study was designed to evaluate the role of traditional and novel risk factors associated with PTDM.

\section{MATERIALS AND METHODS}

The study was approved by the Institutional Ethical Committee dated 18-10-2016, reference number NHH/AEC-CL-2016-096. The prospective observational study was conducted on 30 consecutive renal transplant recipients above the age of 18 years after informed consent. They were followed up to a period of 6 months post renal transplantation. Patients with diagnosis of ESRD due to diabetes mellitus or those who were on anti-diabetic medicines any time prior to transplant were excluded from the study. Demographic details were noted. Details of Pre-transplant risk factors associated with PTDM like native kidney disease, dialysis vintage, family history of diabetes, HCV serology status, BMI, degree of HLA mismatch and type of induction agent used were obtained. During the follow-up, details of posttransplant risk factors associated with PTDM like weight gain, cumulative dose of immunosuppressive medication (oral prednisolone and oral tacrolimus) and rejection episodes were noted. C - reactive protein (CRP) (estimated by nephelometry) levels were used as a marker of pre-transplant inflammatory 
status. Trace elements such as Aluminium, Copper, Selenium and Zinc in the serum were analysed by Inductively coupled Plasma Mass Spectrometry (ICPMS) method. The blood sample for Trace elements was drawn on the day before transplant surgery in live related renal transplant, while the blood sample was drawn prior to the start of induction therapy in deceased donor renal transplant. Other pre-transplant investigations, post-transplant monitoring and follow up were as per the standard of care. In the early post operative period, patients were monitored for trough levels of Serum Tacrolimus (by Enzyme Immunoassay method) and Blood glucose levels at specified time points (Fasting, Post-lunch, Post dinner) by glucometer-based estimation. Trough Tacrolimus levels in the early post-transplant period were noted and categorised as $<10$ or $>10 \mathrm{ng} / \mathrm{ml}$. At the time of discharge, patients were advised to monitor and report Glucometer based Home Self monitoring of blood glucose levels at specified time points (Fasting, post-breakfast, post-lunch and postdinner glucose levels). Oral glucose tolerance test (OGTT) was done at months 1,3 and 6 post renal transplant in patients not diagnosed with PTDM by Home monitoring of blood glucose. Those diagnosed with PTDM were managed by the primary physician/endocrinologist.

\section{Statistical analysis}

Microsoft Excel was used for compiling patient's baseline data. Statistical Software-SPSS version 17.0 was used for descriptive and inferential data analysis. Results on continuous variables are presented on Mean \pm SD and results on categorical variables are presented in number as percent $(\%)$. Correlation between categorical variables was done using Fisher's exact test. Correlation between continuous variables was done using Mann-Whitney test. $p$ value of $<0.05$ was considered statistically significant.

The incidence of PTDM is presented as percentage of the transplant cohort at the end of 6 months. Univariate analysis was performed to determine the significant risk factors associated with development of PTDM. However, a multivariate analysis to determine the impact of various independent risk factors was not feasible due to the small sample size of the study.

\section{RESULTS}

Of the 30 subjects, 28 underwent live related kidney transplant and 2 underwent deceased donor kidney transplant. The average dialysis vintage was 9 months. The mean age of patients in the study was $36.5 \pm 9.9$ years, ranging from 17 years to 53 years. The male to female was ratio of $4: 1$. The native kidney disease was a mix of various causes with chronic glomerulonephritis of unknown aetiology $(76 \%)$ being the most common cause of ESRD. Pre-transplant fasting blood glucose and 2hour post-prandial glucose levels were in the normal range. The demographic details are shown in Table 1.

Table 1: Demographic details of all the renal transplant recipients $(n=30)$

\begin{tabular}{|c|c|c|}
\hline $\begin{array}{c}\text { Parameter } \\
\text { Age (years) }\end{array}$ & Per cent (\%) \\
\hline$<30$ & 8 & 26.7 \\
\hline $30-40$ & 10 & 33.3 \\
\hline$>40$ & 12 & 40 \\
\hline \multicolumn{2}{|c|}{ Sex } \\
\hline Male & 24 & 80 \\
\hline Female & 6 & 20 \\
\hline ADPKD & 1 & 3.3 \\
\hline CGN & 23 & 76.7 \\
\hline IgA Nephropathy & 2 & 6.7 \\
\hline Post-partum cortical necrosis & 1 & 3.3 \\
\hline Renal calculi disease & 1 & 3.3 \\
\hline Obstructive uropathy & 1 & 3.3 \\
\hline \multicolumn{2}{|c|}{ Type of Dialysis } \\
\hline Haemodialysis & 28 & 93.3 \\
\hline Peritoneal dialysis & 1 & 3.3 \\
\hline Pre-emptive transplant & 1 & 3.3 \\
\hline \multicolumn{2}{|c|}{ Type of renal transplant } \\
\hline Live related & 28 & 93.3 \\
\hline Decease donor & 2 & 6.7 \\
\hline
\end{tabular}

At the end of 6 month follow up period, the incidence of PTDM was $36 \%$ in this study. Of the 30 subjects, PTDM occurred in 11 subjects. Post-transplant transient hyperglycemia (PTTH) was found in 17
(56\%) of the patients. Out of the 17 patients with PTTH, 11 patients developed PTDM while 6 patients became euglycemic at the end of 6 months follow up period. In the study population, PTDM was 
diagnosed in $6(54 \%), 3(27 \%)$ and $2(18 \%)$ of the patients at month 1 , month 3 and month 6 post- transplant, respectively. The same has been depicted in Figure 1.

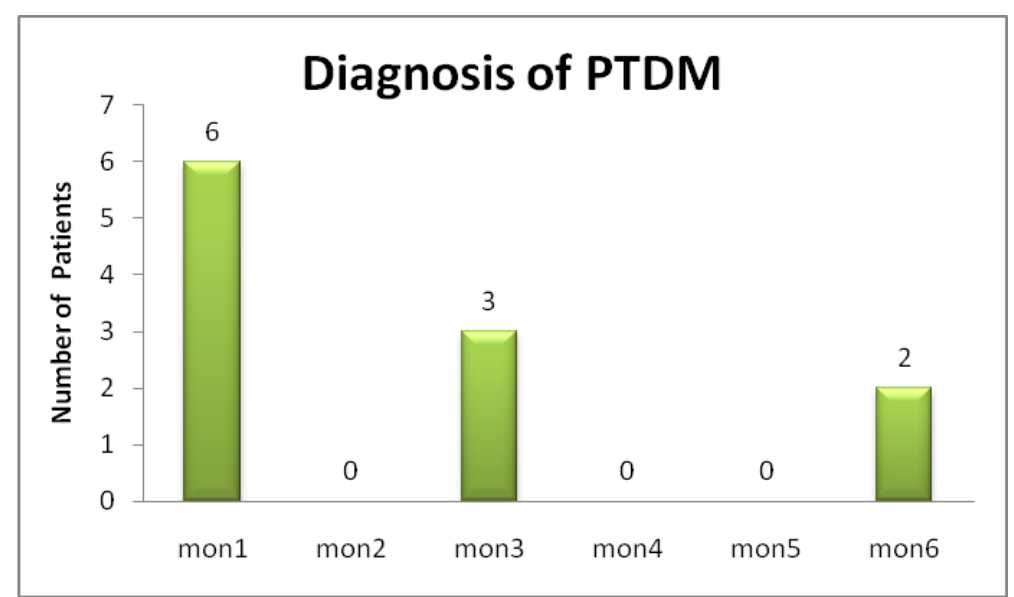

Fig. 1: Number of subjects diagnosed with PTDM during the 6-month follow-up period $(n=11)$

The traditional and novel risk factors for PTDM were categorised as pre-transplant and post-transplant factors. All the subjects were divided into PTDM and non-PTDM cohort for the purpose of analysis. The

Table 2: Pre-transplant risk factors associated with development of PTDM

\begin{tabular}{|c|c|c|c|}
\hline Parameter & Non-PTDM $(n=19)$ & PTDM $(n=11)$ & p value \\
\hline Age (years) & $33.16+9.3$ & $42.36+8.4$ & 0.01 \\
\hline Sex, M: F & $15: 4$ & $9: \overline{2}$ & 0.85 \\
\hline Dialysis vintage (months) & $7.3 \pm 6.2$ & $13.0 \pm 12.8$ & 0.13 \\
\hline \multicolumn{4}{|c|}{ Family history (members) } \\
\hline 0 & $14(73.7 \%)$ & $3(27.2 \%)$ & \multirow[t]{3}{*}{0.007} \\
\hline 1 & $5(26.3 \%)$ & $4(36.4 \%)$ & \\
\hline 2 & $0(0 \%)$ & $4(36.4 \%)$ & \\
\hline BMI (pre-transplant) & $23.7 \pm 5.2$ & $24.5 \pm 5.5$ & 0.73 \\
\hline HCV positive status & $2(10.5 \%)$ & $3(27.3 \%)$ & 0.23 \\
\hline \multicolumn{4}{|c|}{ HLA mismatch } \\
\hline$<3 / 6$ & $8(42.1 \%)$ & $6(54.5 \%)$ & \multirow[t]{2}{*}{0.51} \\
\hline$>3 / 6$ & $11(57.9 \%)$ & $5(45.5 \%)$ & \\
\hline \multicolumn{4}{|c|}{ Induction agent } \\
\hline ATG & $8(42.1 \%)$ & $6(54.5 \%)$ & \multirow[t]{3}{*}{0.51} \\
\hline Basiliximab & $5(26.3 \%)$ & $1(9.1 \%)$ & \\
\hline No Induction (Dexona) & $6(31.6 \%)$ & $4(36.4 \%)$ & \\
\hline
\end{tabular}

The mean age was 42.4 years in the PTDM cohort and 33.5 years in the non-PTDM cohort. Significant proportions of patients with PTDM were above the age of 40 years as shown in Fig. 2.

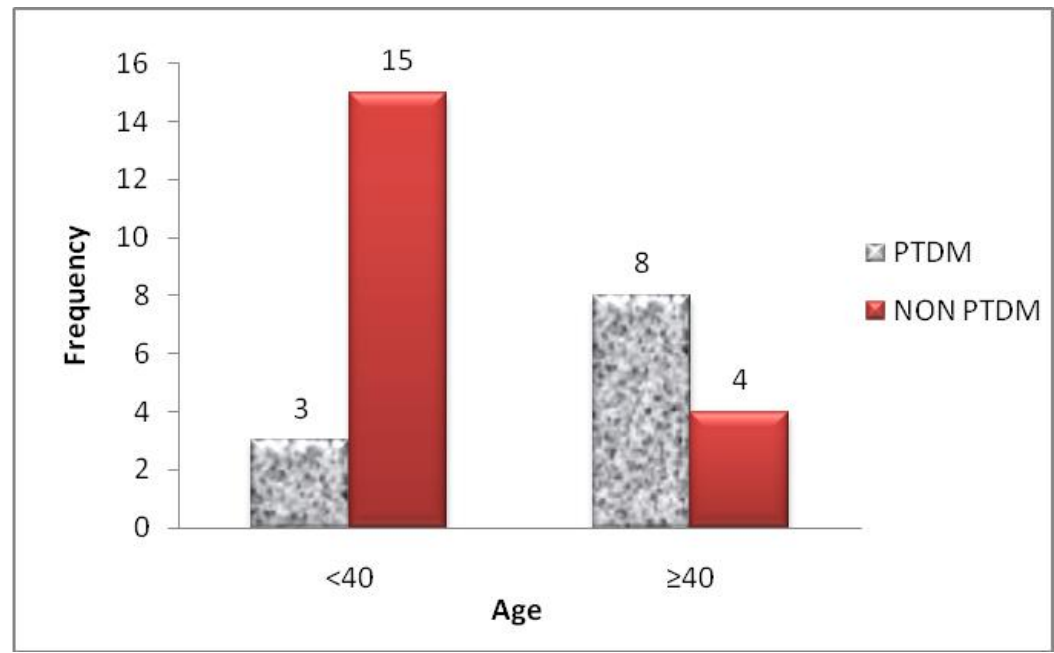

Fig. 2: Proportion of PTDM and non-PTDM subjects above and below 40 years of age 
We looked for presence of diabetes mellitus among the first-degree relatives of the transplant recipients and categorized them as positive history in one or more family members. Positive family history of diabetes mellitus was present in more than two thirds of the subjects who developed PTDM. The BMI was measured at various time points during the study. In the PTDM group, change in BMI over six months was observed. About 15\% subjects moved from normal BMI to Overweight/Obese category with time after renal transplant.

Among the post-transplant risk factors, presence of PTTH had significant association with PTDM. All our patients were on uniform maintenance immunosuppression with triple drug regimen (prednisolone + mycphenolate mofetil + tacrolimus) Post-transplant patients were started on oral prednisolone at $0.4 \mathrm{mg} / \mathrm{kg}$ initially, which was tapered over 4-6 months to reach a long-term maintenance dose of $5 \mathrm{mg} /$ day. Cumulative steroid dose and cumulative tacrolimus dose were similar in both groups. Trough tacrolimus levels and episodes of rejection were higher in the PTDM cohort $(27 \%)$ compared to non-PTDM group (15\%); however, the association was not found to be statistically significant. The post-transplant risk factors associated with PTDM are shown in Table 3.

Table 3: Post-transplant risk factors associated with development of PTDM

\begin{tabular}{|c|c|c|c|}
\hline Parameters & Non PTDM $(\mathbf{n = 1 9})$ & PTDM $(\mathbf{n = 1 1})$ & p value \\
\hline Maximal trough Tacrolimus level & $7.4 \pm 4.4$ & $9.8 \pm 5.1$ & 0.28 \\
\hline PTTH & $6(31.6)$ & $11(100)$ & $\mathbf{0 . 0 0 4}$ \\
\hline Steroid cumulative dose & $2218 \pm 425.3$ & $2242 \pm 427.0$ & 0.96 \\
\hline CNI cumulative dose & $935.6 \pm 317.3$ & $792.8 \pm 340.1$ & 0.13 \\
\hline Rejection episodes & $3(15.8)$ & $3(27.3)$ & 0.51 \\
\hline
\end{tabular}

Trace element deficiency and Pre-transplant Creactive protein (CRP) were considered as a novel risk factor for development of PTDM. Among the trace elements, pre-transplant blood levels of Magnesium ( $\mathrm{Mg}$ ), Aluminium ( $\mathrm{Al})$, Selenium (Se), Copper $(\mathrm{Cu})$ and Zinc $(\mathrm{Zn})$ were assessed. Trace element levels were categorised as normal, high or low. The pre-transplant levels of selenium were normal in all subjects. Aluminium levels were high in $10 \%$ of the subjects. Low levels of Magnesium and Copper were seen in $10 \%$ and $16 \%$ of the subjects respectively. Low level of Zinc was found in $66.7 \%$ of subjects. The proportion of subjects among the total study population, with various trace element levels pre transplant has been depicted in Figure 3 .

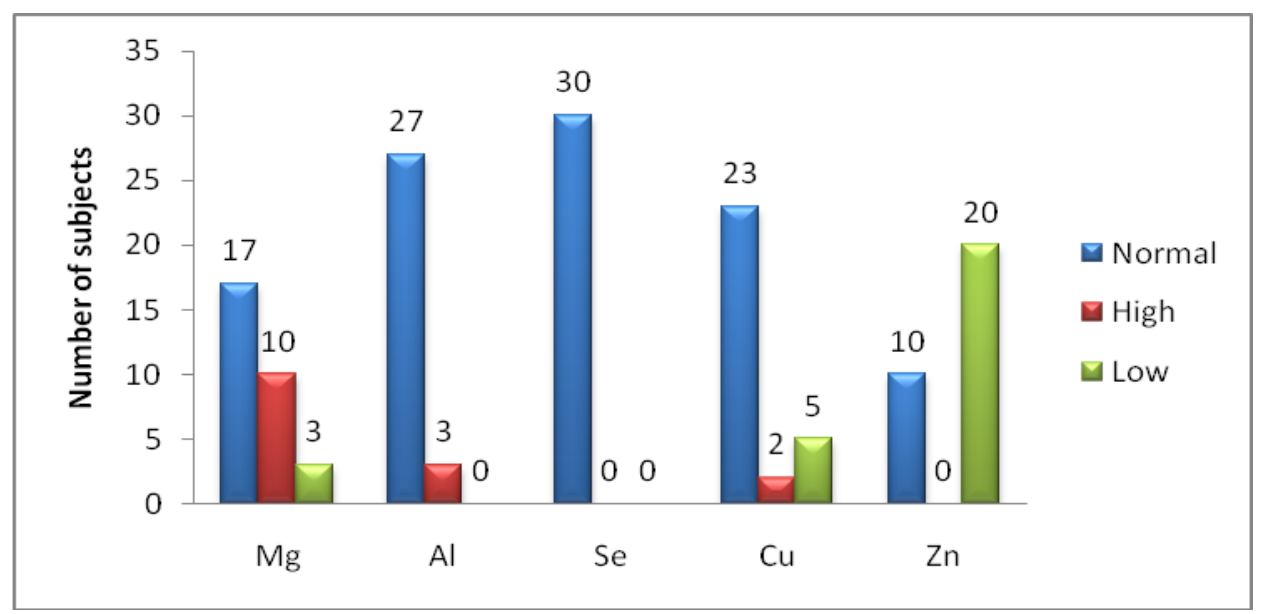

Fig. 3: Distribution of subjects with normal, high or low trace element levels Pre-transplant $(n=30)$

Pre transplant CRP level ranged from $0.2 \mathrm{mg} / \mathrm{L}-14.6$ $\mathrm{mg} / \mathrm{L}$ with the mean CRP level being $2.69 \mathrm{mg} / \mathrm{L}$. The levels were similar between both groups and did not have an association with development of PTDM. Among the trace elements, low levels of magnesium, copper and zinc were seen in both the PTDM and non
PTDM groups. However, only zinc deficiency was found to have a significant association with the occurrence of PTDM. The proportion of subjects with trace element deficiency in both PTDM and non PTDM groups with significant association is shown in Table 4.

Table 4: Novel risk factors (Pre transplant CRP and trace elements) associated with PTDM

\begin{tabular}{|c|c|c|c|}
\hline Parameter & Non-PTDM (n=19) & PTDM (n=11) & p value \\
\hline CRP (mg/L) & $2.8 \pm 3.4$ & $2.4 \pm 1.7$ & 0.77 \\
\hline Trace element deficiency & & & \\
Magnesium & $1(5.3 \%)$ & $2(18.2 \%)$ & 0.45 \\
Zinc & $10(52.6 \%)$ & $10(90.9 \%)$ & $\mathbf{0 . 0 3}$ \\
Copper & $3(15.8 \%)$ & $2(18.2 \%)$ & 0.89 \\
\hline
\end{tabular}




\section{DISCUSSION}

Globally, the reported incidence of PTDM ranges from $4-30 \%$ (2). Among the Indian studies, the incidence of PTDM appears to be slightly, higher ranging between $21-34 \%(3-5)$ as compared to the worldwide data (6). Differences in studies with regards to use of standardized definitions, length of follow-up, intensity of routine screening, inclusion of OGTT and glycated hemoglobin (HbAlc) testing have had an impact on incidence estimates. The incidence of Post Transplant Diabetes Mellitus (PTDM) in our cohort of renal transplant recipients was $36.6 \%$, which is similar to other studies which have reported a higher incidence of PTDM in subjects of Asian ethnicity.

Among the known traditional risk factors, higher age and positive family history of diabetes mellitus had a strong association in the occurrence of PTDM. Other risk factors like gender, native kidney disease, type of donation, HCV infection, degree of HLA mismatch, type of induction agent and cumulative dose of steroids/CNI's did not show association with development of PTDM. Among the novel risk factors, while pre-transplant inflammatory status did not confer significance, Trace element deficiency was associated with development of PTDM.

Till date, higher age has been found to be the independent risk factor associated with PTDM. In a study by Cosio et al., age above 45 years was shown to be associated with 2.9 times higher risk of developing PTDM (7). In another study on Canadian population, the incidence of PTDM was found to increase by $50 \%$ for every 10 -year increase in age (8). Among those who developed PTDM in our study, $70 \%$ of the patients were above the age of 40 years $(p<0.01)$. Similar to other studies $(3,4,5)$ higher the age, higher the risk for PTDM is clearly evident in our study.

Similar to type 2 diabetes mellitus in the general population, genetic and environmental factors have been suggested to play a role in the development of PTDM. Presence of family history of diabetes mellitus has been implicated to have 2 to 7 times increased risk of developing PTDM $(9,10)$. In our study, family history of diabetes mellitus was present in $70 \%$ of the patients who developed PTDM. We looked for presence of diabetes mellitus among the first-degree relatives of the transplant recipients and categorized them as positive history in one or more family members. Similar to previous studies, we found that presence of DM in one or more family members was associated with significantly higher risk of PTDM ( $\mathrm{p}<0.007)$.

Obesity has detrimental effects on transplantation outcomes for a variety of reasons, one of them being risk for PTDM. In our study, the pre-transplant BMI was similar between both the PTDM and non-PTDM groups. Even at 6-months post-transplant, the BMI between both groups were not very different. Among those who developed PTDM, 27\% of patients were in the overweight/obese category pre-transplant. At 6month post-transplant, the patients in the overweight/obese category increased to $45 \%$. About $15 \%$ of patients had increased their BMI over a 6month period post-transplant. However, the change in BMI did not confer statistical significance.

The role of steroids in contributing to PTDM among renal transplant recipients was first described by Starlz in the year 1964 (2). However, the results of a double-blind randomized trial indicated that early corticosteroid withdrawal had limited impact in reducing PTDM when compared to low-dose prednisone (11). We calculated the cumulative dose of oral prednisolone over 6 months post-transplant to assess the dose dependent risk associated with PTDM. However, the cumulative dose of steroids used was comparable between the PTDM and nonPTDM groups. This lack of association between steroid use and PTDM clearly indicates that occurrence of PTDM is multi-factorial influenced by changes in patient risk profiles and changes in immunosuppressive medications.

Of the various inflammatory markers like Adiponectin, CRP, TNF-alpha and IL, high TNFalpha levels were found to be associated with PTDM (12). We looked at pre-transplant CRP levels as a marker of inflammatory status. In our study the pretransplant CRP levels were within the normal range in both the groups. Though CRP levels were found to be higher in the PTDM group, it was not statistically significant.

Considering hypomagnesemia as a risk factor for PTDM, some studies have looked at the effect of magnesium supplements in early post-transplantation period. High doses of magnesium oxide have had a beneficial, but minor effect on glycemic control (13). In our study, pre-transplant serum magnesium levels were in the normal range in 50\%, high in $30 \%$ and low only in $10 \%$ of patients in the entire cohort. However, among those who developed PTDM, 18\% patients had hypomagnesaemia as compared to 5\% in those who did not develop PTDM. Though the proportion of patients with hypomagnesaemia was higher in the PTDM group, we found no statistical significance.

Among the trace elements, deficiency of Zinc and Copper as possible risk factor for diabetes mellitus in the general population have been studied extensively. Zinc is a trace element and an essential component of more than 300 catalytic enzymes in the body $(14,15)$. The Zinc content in pancreatic $\beta$-cells is among the highest of the body (16) and acts as a co-factor in the synthesis, storage and secretions of insulin by pancreatic tissue, also accounting for the conformation integrity of insulin in its hexameric 
crystalline structure. Different Zinc Transporter (ZnTs) including ZnT8, ZnT3, and ZIP6 have specific roles in insulin pathway (17). Observations of hyperzincuria, hypozincemia and zinc malabsorption in patients with diabetes, indicate their role in the pathogenic mechanism. Many of the complications of diabetes are related to the decrease in intracellular zinc and zinc dependent antioxidant enzymes. A study by Praveena et al (18) has shown that serum zinc levels in the diabetic patients was found to be significantly lower than that of the control group. Low levels of Zinc have been demonstrated in patients of type 2 diabetes mellitus consistently (1921). In our study, of the entire cohort, Zinc level was in the normal range in $33 \%$ of patients and was low in $66 \%$ of patients prior to transplant. In those who developed PTDM, 90\% patients had low zinc as compared to $50 \%$ in those who did not develop PTDM $(\mathrm{p}<0.03)$. Our study shows that Zinc deficiency was associated with PTDM even in the transplant setting. We found low Copper levels in $16 \%$ of patients of the entire cohort. Apart from Zinc, other trace elements like copper, selenium and aluminium did not seem to have an impact on PTDM in our study. To our knowledge, this study remains unique since there have been no studies looking at trace element deficiency in a cohort of renal transplant recipients. Till date, studies on trace element deficiency and diabetes have been done in the general population. Though there are no guidelines for zinc measurement or supplementation in patients with diabetes, zinc supplementation has been observed in several studies to positively affect $\mathrm{HbA1c}$, glucose and lipid levels with potential for decreasing oxidative stress and protection from renal damage (22-24). Along with strict glycemic control and management of known risk factors, repletion of trace elements may be an effective therapeutic intervention in preventing progression of diabetes and its associated complications.

Post-transplant transient hyperglycemia (PTTH) is a recently introduced terminology during the consensus meeting on PTDM in 2014. In the available literature, prior to 2014, PTTH has been referred to as in-patient hyperglycemia or early onset hyperglycemia or hyperglycemia requiring insulin therapy temporarily. In a study by Chakkera et al., (25) $48 \%$ of the patients who had in-patient hyperglycemia developed PTDM. Cosio et al., reported that hyperglycemia during the first week after transplantation is the strongest predictor of PTDM at 1 year (7). In a study by Tapia (26) on 163 renal transplant recipients, 16 $\%$ developed PTDM. Early hyperglycemia was observed in $60 \%$ of the subjects. In our study we found $56 \%$ of patients to have had PTTH. In those who developed PTDM, all the patients had PTTH. In those who did not develop PTDM, 36\% patients had PTTH. Presence of PTTH was found to have significant association with occurrence of PTDM $(p<$
0.004). Our study results correlate very well with previous studies where in-patient and early hyperglycemia post-transplant prove to be a major risk factor for future PTDM. With the new definition of PTTH in place, studies with new design and testing strategies in high-risk patients may be the next logical step in prevention of PTDM.

\section{CONCLUSION}

We found higher age, zinc deficiency and posttransplant transient hyperglycemia to be significant risk factors associated with future development of PTDM. The inclusion of PTDM as a pre-specified endpoint will help to better understand not only the most important contributing risk for PTDM but also identify those interventions that are most likely to result in a lower incidence of PTDM. We suggest further studies to look at novel risk factors associated with development of PTDM. Along with strict glycemic control and management of known risk factors, repletion of trace elements may be an effective therapeutic intervention in preventing progression of diabetes and its associated complications.

\section{ACKNOWLEDGMENT}

Authors acknowledge the support of the patients for agreeing to participate in the study and follow-up as per protocol.

\section{CONFLICT OF INTEREST}

Authors declare that there is no conflicting interests.

\section{REFERNCES}

1. Sharif, A., Hecking, M., de Vries, A. P., Porrini, E., Hornum, M., Rasoul, R. S., et al., Proceedings from an international consensus meeting on posttransplantation diabetes mellitus: recommendations and future directions. Am J Transplant. 2014 Sep; 14(9): 1992-2000.

2. Pham, P. T., Pham, P. M., Pham, S. V., Pham, P. A., Pham, P. C. New onset diabetes after transplantation (NODAT): an overview. Diabetes Metab Syndr Obes. 2011; 4: 175-186.

3. Lakshminarayana, G. R., Sheetal, L. G., Anil, M. New Onset Diabetes after Renal Transplantation (NODAT): Prevalence, Risk Factors and Treatment. JMSCR. 2016 Jan; 04: 8969-8975.

4. Saboo, D., Shah, P. R., Goplani, K. R., Feroz, A., Gumber, M., Vanikar, A. V., et al., Posttransplant diabetes mellitus: a single-center study. Transplant Proc. 2008 May; 40(4): 1111-1113.

5. Patel, D. D., Modi, K. P., Patel, A. K., Chaudhary, V. New onset of diabetes mellitus in Indian renal transplant recipient-a retrospective study. International Journal of Pharmacy and Pharmaceutical Sciences. 2015; 7(11): 228232.

6. Montori, V. M., Basu, A., Erwin, P. J., Velosa, J. A., Gabriel, S. E., Kudva, Y. C. Posttransplantation diabetes: a systematic review of the literature. Diabetes Care. 2002 Mar;25(3): 583-592.

7. Cosio, F. G., Pesavento, T. E., Osei, K., Henry, M. L., Ferguson, R. M. Post-transplant diabetes mellitus: increasing incidence in renal allograft recipients transplanted in recent years. Kidney Int. $2001 \mathrm{Feb}$; 59(2): 732-737.

8. Gourishankar, S., Jhangri, G. S., Tonelli, M., Wales, L. H., Cockfield, S. M. Development of diabetes mellitus 
following kidney transplantation: a Canadian experience. Am J Transplant. 2004 Nov; 4(11): 1876-1882.

9. Martínez, C. A., Hernández, M. D., Pascual, J., Morales, J. M., Marcen, R., Errasti, P., et al., Detection and treatment of post kidney transplant hyperglycemia: a Spanish multicenter cross-sectional study. Transplant Proc. 2005 Nov; 37(9): 3813-3816.

10. Davidson, J., Wilkinson, A., Dantal, J., Dotta, F., Haller, H., Hernández, D., et al., New-onset diabetes after transplantation: 2003 International consensus guidelines. Proceedings of an international expert panel meeting. Barcelona, Spain, 19 February 2003. Transplantation. 2003 May 27; 75(10 Suppl): SS3- SS24.

11. Pirsch, J. D., Henning, A. K., First, M. R., Fitzsimmons, W., Gaber, A. O., Reisfield, R., et al., New-onset diabetes after transplantation: results from a double-blind early corticosteroid withdrawal trial. Am J Transplant. 2015 Jul; 15(7): 1982-1990.

12. Cantarin, M. P., Keith, S. W., Lin, Z., Doria, C., Frank, A. M., Maley, W. R., et al., Association of inflammation prior to kidney transplantation with post-transplant diabetes mellitus. Cardiorenal Med. 2016 Aug; 6(4): 289-300.

13. van Laecke, S., Nagler, E. V., Taes, Y., van Biesen, W., Peeters, P., Vanholder, R. The effect of magnesium supplements on early post-transplantation glucose metabolism: a randomized controlled trial. Transpl Int. 2014 Sep; 27(9): 895-902.

14. Jansen, J., Karges, W., Rink, L. Zinc and diabetes - Clinical links and molecular mechanisms. J Nutr Biochem. 2009; 20: 399-417.

15. Khan, A. R., Awan, F. R. Metals in the pathogenesis of type 2 diabetes. J Diabetes Metab Disord. 2014; 13: 16.

16. Chimienti, F., Favier, A., Seve, M. ZnT-8, a pancreatic beta-cell-specific zinc transporter. Biometals 2005; 18: 313-317.

17. Rungby, J. Zinc, zinc transporters and diabetes. Diabetologia. 2010; 53: 1549-1551.

18. Praveena, S., Pasula, S., Sameera, K. Trace elements in diabetes mellitus. J Clin Diagn Res. 2013 Sep; 7(9): 18631865.

19. Terrés, M. C., Navarro, A. M., Martín, L. F., López, G., Serrana, H., Pérez, V. V., et al., Serum zinc and copper concentrations and $\mathrm{Cu} / \mathrm{Zn}$ ratios in patients with hepatopathies or diabetes. J Trace Elem Med Biol. 1998 Mar; 12(1): 44-49.

20. Basaki, M., Saeb, M., Nazifi, S., Shamsaei, H. A. Zinc, copper, iron, and chromium concentrations in young patients with type 2 diabetes mellitus. Biol Trace Elem Res. 2012 Aug; 148(2): 161-164.

21. Devi, T. R., Hijam, D., Dubey, A. Study of serum zinc and copper levels in type 2 diabetes mellitus. International Journal of Contemporary Medical Research. 2016; 3(4): 1036-1040.

22. Gunasekara, P., Hettiarachchi, M., Liyanage, C., Lekamwasam, S. Effects of zinc and multimineral vitamin supplementation on glycemic and lipid control in adult diabetes. Diabetes Metab Syndr Obes. 2011; 4: 53-60.

23. Foster, M., Petocz, P., Samman, S. Effects of zinc on plasma lipoprotein cholesterol concentrations in humans: A meta-analysis of randomised controlled trials. Atherosclerosis. 2010; 210: 344-352.

24. Jayawardena, R., Ranasinghe, P., Galappatthy, P., Malkanthi, R., Constantine, G., Katulanda, P. Effects of zinc supplementation on diabetes mellitus: A systematic review and meta-analysis. Diabetol Metab Syndr. 2012; 4: 13.

25. Chakkera, H. A., Knowler, W. C., Devarapalli, Y., Weil, E. J., Heilman, R. L., Dueck, A., et al., Relationship between inpatient hyperglycemia and insulin treatment after kidney transplantation and future new onset diabetes mellitus. Clin J Am Soc Nephrol. 2010 Sep; 5(9): 1669-1675.

26. Maldonado, F., Tapia, G., Ardiles, L. Early hyperglycemia: a risk factor for posttransplant diabetes mellitus among renal transplant recipients. Transplant Proc. 2009 Jul-Aug; 41(6): 2664-2667. 